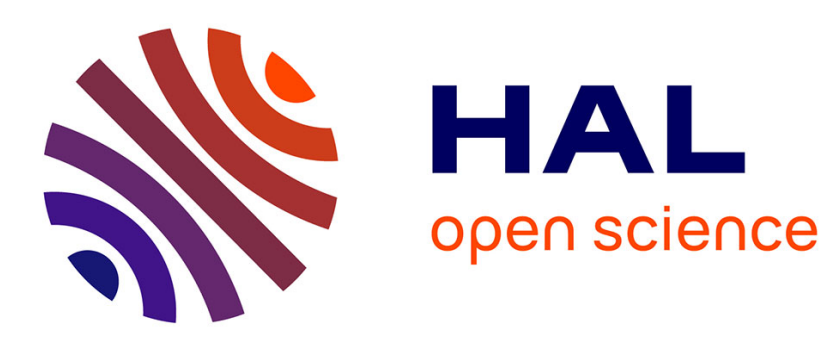

\title{
Stability analysis of networked control systems with asynchronous sampling and input delay
}

\author{
Alexandre Seuret
}

\section{To cite this version:}

Alexandre Seuret. Stability analysis of networked control systems with asynchronous sampling and input delay. ACC 2011 - American Control Conference, Jun 2011, San Francisco, Californie, United States. pp.6. hal-00578771

\section{HAL Id: hal-00578771 \\ https://hal.science/hal-00578771}

Submitted on 22 Mar 2011

HAL is a multi-disciplinary open access archive for the deposit and dissemination of scientific research documents, whether they are published or not. The documents may come from teaching and research institutions in France or abroad, or from public or private research centers.
L'archive ouverte pluridisciplinaire HAL, est destinée au dépôt et à la diffusion de documents scientifiques de niveau recherche, publiés ou non, émanant des établissements d'enseignement et de recherche français ou étrangers, des laboratoires publics ou privés. 


\title{
Stability analysis of networked control systems with asynchronous sampling and input delay
}

\author{
Alexandre Seuret \\ alexandre.seuret@gipsa-lab.inpg.fr \\ NeCS Team, Department of Automatic Control, \\ GIPSA-Lab-CNRS UMR 5216, Grenoble, France
}

\begin{abstract}
This article proposes a novel approach to assess the stability of linear systems with delayed and sampled-data inputs. The paper considers both asynchronous sampling and input delay based on an extension of existing results on the stability of sampled-data systems to the case where a delay is introduced in the control loop. The proposed method provides easy tractable sufficient conditions for asymptotic stability of sampled-data systems under asynchronous sampling and transmission delays. The period and delay-dependent conditions are expressed using computable linear matrix inequalities. Several examples show the efficiency of the stability criteria.
\end{abstract}

\section{INTRODUCTION}

In the last decades, a large attention has been taken to Networked Control Systems (NCS) (see [7], or [22]). Such systems are controlled systems containing several distributed plants which are connected through a communication network. In such applications, one has to check the robustness of a control law with respect to the additional dynamics introduced by the communication networks. Among these dynamics, this article focuses on the influence of transmission delay and asynchronous samplings. The transmission of a data packet through a network can not be achieved instantaneously. Transmission delays are unavoidably introduced. Those delays may lead to instability [18]. It is thus an important issue to develop robust stability criteria with respect to transmission delays and asynchronous samplings.

Sampled-data systems have extensively been studied in the literature [1], [3], [5], [23], [24] and the references therein. It is now reasonable to design controllers which guarantee the robustness of the solutions of the closed-loop system under periodic samplings. However the case of asynchronous samplings still leads to several open problems such that the guarantee of stability whatever the sampling period lying in an interval. Recently, several articles drive the problem of time-varying periods based on a discrete-time approach, [8], [16], [21]. Note that the discrete-time approaches do not fit with the case of uncertain systems or systems with timevarying parameters. Recent papers considered the modelling of continuous-time systems with sampled-data control in the form of continuous-time systems with delayed control input. In [3], a Lyapunov-Krasovskii approach is introduced.

This research is supported by FeedNetBack project, FP7- ICT-2007-2: http://www.feednetback.eu/.
Improvements are provided in [5], [13], using the small gain theorem and in [14] based on the analysis of impulsive systems. These approaches are very relevant because they deal with time-varying sampling periods and with uncertain systems (see [3] and [14]). Nevertheless, these sufficient conditions are still conservative. This means that the conditions obtained by continuous-time approaches are not able to guarantee asymptotic stability whereas the system is stable. Recently several authors [2], [19] refine those approaches and obtain tighter conditions.

When transmission delays are introduced in the control loop, the problem becomes more complex. It is indeed well known that delays require a more accurate analysis since the time-delay systems are of infinite dimension [6], [18]. Several articles have been provided to cope with the stability of NCS under sampling and transmission delays. In [3], [11], [15], stability conditions of systems under asynchronous sampling and transmission delays are presented. However those conditions are still conservative and require improvements. In the present article, we provide a novel method to assess asymptotic stability of such systems. The conditions are presented as an extension of [20] to the case of time-varying transmission delays. This problem of NCS is hybrid since we consider a continuous time model of the plant and a discrete-time communication. Thus an important improvement addressed inthis article consists in employing the discrete-time Lyapunov theorem to continuous-time modelling of sampled-data systems with delays. The main contribution of this paper is to consider separately the two types of delays.

This article is organized as follows. The next section formulates the problem. Section III exposes the novel stability criteria based on the discrete-time Lyapunov theorem. Then,in Sections IV and V, asymptotic stability criteria sampled-data systems are exposed to cope respectively with the cases of constant and time-varying input delays. Some examples are provided in Section VI which shows the efficiency of the method.

Notations. Throughout the article, for a n-dimensional state vector $x$ and a non-negative delay $h, x_{t}$ denotes $a$ function such that $x_{t}(\theta)=x(t-\theta)$ for all $\theta \in[-h, 0]$ The set of such functions $x_{t}$ is denoted as $\mathbb{K}^{h}$. The sets $\mathbb{R}^{+}, \mathbb{R}^{n \times n}$ and $\mathbb{S}^{n}$ denote respectively the set of positive scalar, the set of $n \times n$ matrices and the set of symmetric 


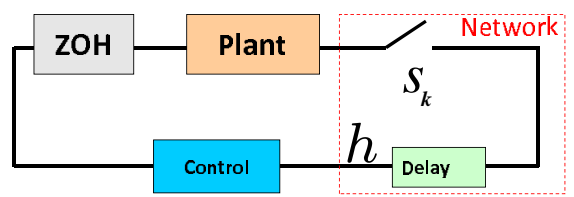

Fig. 1. Control loop of Networked control systems under transmission and sampling delays

matrices of $\mathbb{R}^{n \times n}$. The superscript ' $T$ ' stands for the matrix transposition. The notation $P>0$ for $P \in \mathbb{S}^{n}$ means that $P$ is positive definite. The symbols $I$ and 0 represent the identity and the zero matrices of appropriate dimension.

\section{PROBLEM FORMULATION}

\section{A. System definition}

Consider the linear system with a sampled and delayed input as shown in Figure 1:

$$
\dot{x}(t)=A x(t)+B u(t)
$$

where $x \in \mathbb{R}^{n}$ and $u \in \mathbb{R}^{m}$ represent the state variable and the input vector. The matrices $A$ and $B$ are constant and of appropriate dimension. As in the situation of networked control systems, the control input $u$ is affected by the networked communication. In this paper it is assumed that the network induces a time-varying transmission delay $h$ and a sampling of the transmitted signal. The control law is a piecewise-constant static state-feedback of the form:

$$
u(t)=K x\left(s_{k}\right), s_{k}+h\left(s_{k}\right) \leq t<s_{k+1}+h\left(s_{k+1}\right),
$$

where the gain $K$ in $\mathbb{R}^{n \times m}$ is given and where $0=s_{0}<$ $s_{1}<\ldots<s_{k}<\ldots$ represent the sampling instants from the sensors. The sequence of $\left\{s_{k}\right\}_{k \geq 0}$ is strictly increasing and goes to infinity as $k$ increases. The transmission delay $h(t)$ is assumed to be constant or time-varying and such that

$$
\forall t, \quad h(t) \in\left[h_{1} h_{2}\right], \quad \epsilon_{1} \leq \dot{h}(t) \leq \epsilon_{2}<1,
$$

where $0 \leq h_{1}<h_{2}$ and $\epsilon_{1}<\epsilon_{2}$. In order to simplify the notation, $h_{k}=h\left(s_{k}\right)$ is introduced. Denote $t_{k}=s_{k}+h_{k}$. These instants $t_{k}$ represent the instants where the control input is updated. The condition $\dot{h}<1$ ensures that the sequence of $t_{k}$ 's is strictly increasing. The closed loop system is thus rewritten as

$$
\dot{x}(t)=A x(t)+A_{d} x\left(t_{k}-h_{k}\right), \quad t_{k} \leq t<t_{k+1},
$$

where $A_{d}=B K$. Assume that there exists two positive scalars $T_{1}<T_{2}$ such that the difference between two successive sampling instants $T_{k}=s_{k+1}-s_{k}$ satisfies

$$
\forall k \geq 0, \quad 0 \leq T_{1} \leq T_{k} \leq T_{2}
$$

Then the length of the sampling interval in the actuator, $\bar{T}_{k}=$ $t_{k+1}-t_{k}$, satisfies

$\bar{T}_{k}=t_{k+1}-t_{k}=s_{k+1}-s_{k}+h_{k+1}-h_{k}=T_{k}+h_{k+1}-h_{k}$.

This show the influence of a time-varying delay in the synchrony of the sampling at the actuator. This means that

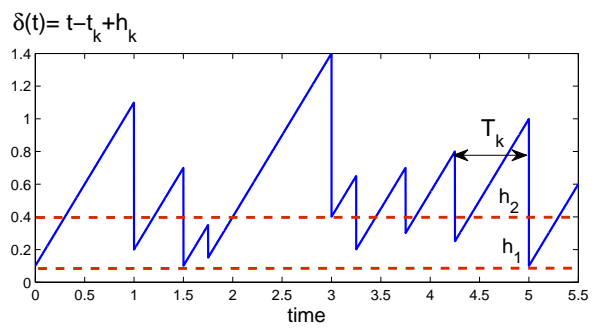

Fig. 2. Examples of a delay generated by a transmission delay $h_{k}$ bounded by $h_{1}$ and $h_{2}$ and an asynchronous sampling of periods $T_{k}$

even if the sampling at the sensor is periodic, the sampling at the actuator becomes asynchronous. The chronological order of the control values is ensured by the positivity of $\bar{T}_{k}$. Finally the following bounds of the sampling period of the actuator is bounded by:

$$
\forall k \geq 0 \leq \bar{T}_{1} \leq \bar{T}_{k} \leq \bar{T}_{2}
$$

where $\bar{T}_{1}=T_{1}-\left(h_{2}-h_{1}\right)$ and $\bar{T}_{2}=T_{2}+\left(h_{2}-h_{1}\right)$. Note that the sampling period at the sensor and at the actuators are equal if the transmission delay is constant. In other words, $\bar{T}_{1}=T_{1}$ and $\bar{T}_{2}=T_{2}$.

Several authors investigated in guaranteeing the stability of such systems. In [3], a continuous-time approach to model sampled-data systems was developed. It allows assimilating sampling effects as the ones of a particular delay. We will further consider (3) as a linear system with uncertain and bounded delay $\delta(t)=t-t_{k}+h_{k}$. An example of such delays is presented in Figure 2. In [3], [12] or [11], the authors propose an aggregated delay formulation. They develop stability criteria which take into account the delay $\delta$. However they did not consider the different natures of the transmission and the sampling delay. More especially the additional characteristic of sampled delay which is $\dot{\delta}=1$ has not been included and thus leads to conservative conditions.

The discrete-time modelling of such systems is obtained by integrating the differential equation (3) over the interval $\left[t_{k}, t_{k}+\tau\right]$, for any $\tau$ in $\left[0, \bar{T}_{k}\right]$,

$$
\begin{aligned}
& x\left(t_{k}+\tau\right)=\tilde{A}(\tau) x\left(t_{k}\right)+\tilde{A}_{d}(\tau) x\left(t_{k}-h_{k}\right), \\
& \tilde{A}(\tau)=e^{A \tau}, \quad \tilde{A}_{d}(\tau)=\int_{0}^{\tau} e^{A(\tau-\theta)} d \theta B K .
\end{aligned}
$$

This equality leads naturally to the introduction of a novel notation. Define, for all integer $k$, the function $\chi_{k}^{h_{2}}:\left[0, T_{k}\right] \times$ $\left[-h_{2}, 0\right] \rightarrow \mathbb{R}^{n}$ such that for all $\tau$ in $\left[0, T_{k}\right]$ and all $\theta$ in $\left[-h_{2}, 0\right], \chi_{k}(\tau, \theta)=x\left(t_{k}+\tau+\theta\right)$. The set $\mathbb{K}_{\bar{T}}^{h_{2}}$ represents the set of functions defined by $\chi_{k}^{h_{2}}$ as the set of continuous functions from $[0, \bar{T}] \times\left[-h_{2}, 0\right]$ to $\mathbb{R}^{n}$ where $\bar{T}$ is the upper-bound of the $T_{k}$ 's in (4).

Taking $\tau=t_{k+1}-t_{k}$ in (6), a recurrence equation is obtained of the form $x_{k+1}=\tilde{A}\left(T_{k}\right) x_{k}+\tilde{A}_{d}\left(T_{k}\right) x\left(t_{k}-h_{k}\right)$. In the particular situation of a delay $h_{k}$ equal to $t_{k}-t_{k-i}$ where $i$ is an integer, the model can be rewritten as $x_{k+1}=$ $\tilde{A}\left(T_{k}\right) x_{k}+\tilde{A}_{d}\left(T_{k}\right) x_{k-i}$. In this situation, several stability conditions can be seen in the literature (see for instance [4]) based on the increment of a Lyapunov function. However, 
in practice there is no guarantee that the instant $t_{k}-h_{k}$ corresponds exactly to a previous sampling instant. Thus there is a need to introduce novel stability conditions to cope with this type of discrete-time systems.

In this paper, the aggregated delay $\delta$ representing the effect of the transmission and the sampling delays is split into two parts. In this paper, a novel method to assess stability of systems subject to this type of delay is proposed. The main idea is to consider separately the two types delays. To do so, the stability conditions are based on the discrete-time Lyapunov Theorem and leads to less conservative necessary conditions.

\section{MAIN RESULT}

This section is motivated by the difference between the discrete and continuous-time Lyapunov Theorems. As the problem of sampled-data systems is at the boundary of the discrete and the continuous-time theories, it is important to put in clear the difference between them. More especially, the main idea of this section consists in developing a novel stability criterion for systems, taken in a continuous-time model, using the discrete-time Lyapunov Theorem.

Theorem 1: Let $V: \mathbb{K}^{h_{2}} \rightarrow \mathbb{R}^{+}$be a functional for which there exist real numbers $0<\mu_{1}<\mu_{2}$ and $p>0$ such that

$$
\forall\left(x_{t}\right) \in \mathbb{K}, \quad \mu_{1}\left|x_{t}(0)\right|^{p} \leq V\left(x_{t}\right) \leq \mu_{2}\left|x_{t}\right|^{p} .
$$

The two following statements are equivalent.

(i) $\quad \forall k \geq 0, \quad \Delta V(k)=V\left(x_{t_{k+1}}\right)-V\left(x_{t_{k}}\right)<0$;

(ii) There exists a continuous functional $\mathcal{V}: \mathbb{R} \times \mathbb{K}_{\bar{T}}^{h_{2}} \rightarrow$ $\mathbb{R}$, differentiable over all intervals of the form $\left[t_{k} t_{k+1}[\right.$ which satisfies

$$
\forall k \geq 0, \quad \mathcal{V}\left(T_{k}, \chi_{k}^{h_{2}}\right)=\mathcal{V}\left(0, \chi_{k}^{h_{2}}\right)
$$

and such that, for all $k>0$ and for all $t$ in $\left[t_{k} t_{k+1}\right]$, the following inequality holds

$$
\dot{\mathcal{W}}\left(\boldsymbol{\tau}, \chi_{k}^{h_{2}}\right)<0
$$

where $\tau=\tau(t)=t-t_{k}$ and

$$
\dot{\mathcal{W}}\left(\boldsymbol{\tau}, \chi_{k}^{h_{2}}\right)=\frac{d}{d t}\left\{\left[V\left(x_{t}\right)+\mathcal{V}\left(\boldsymbol{\tau}, \chi_{k}^{h_{2}}\right)\right]\right\} \text {. }
$$

Moreover, if one of these two statements is satisfied, the solutions of system (3) are asymptotically stable.

Proof: Consider a positive integer $k$ and $t \in\left[t_{k}, t_{k+1}\right]$. Assume $(i i)$ is satisfied. Integrating $\dot{\mathcal{W}}$ over the interval $\left[t_{k} t_{k+1}\right.$ [ and assuming that (8) holds, this directly implies $\Delta V(k)<0$ and that $(i)$ holds.

Assume now that $(i)$ is satisfied. Inspired by Lemma 2 in [17], consider the functional $\mathcal{V}\left(\tau, \chi_{k}^{h_{2}}\right)=-V\left(x_{t}\right)+$ $\tau / T_{k} \Delta V(k)$. Indeed, $\mathcal{V}$ is a functional since it is expressed with respect to $\Delta V(k)$ which depends on the function $\chi_{k}^{h_{2}}$. By simple computations, it is easy to obtain that this functional satisfies (8) and that $\dot{\mathcal{W}}\left(\tau, \chi_{k}\right)=\Delta V(k)$. Thus, $\dot{\mathcal{W}}$ has the same sign as $\Delta V(k)$. This proves the equivalence between $(i)$ and $(i i)$.

From the discrete-time Lyapunov theorem, the equilibrium of the discrete-time system is asymptotically stable.

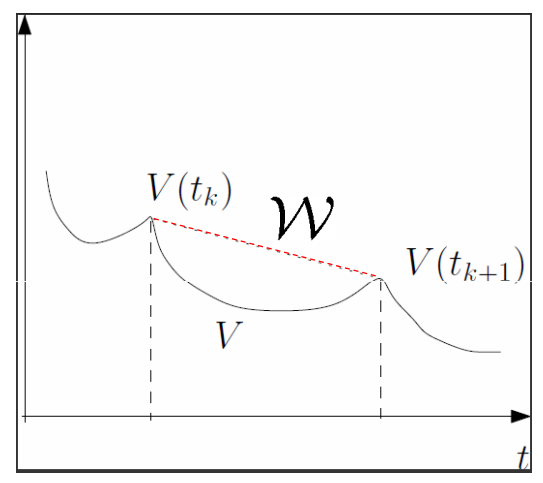

Fig. 3. Illustration of the proof of Theorem 1

The end of the proof consists in ensuring that the solutions of the continuous-time system are not diverging within a sampling period. Consider any $s \in\left[t_{k}, t_{k+1}\right]$. From (7), it follows that $V\left(x_{s}\right)<\lambda_{2}\left|x_{s}\right|$. From (6), the following equality holds

$$
x(s)=\tilde{A}(\tau(s)) x\left(t_{k}\right)+\tilde{A}_{d}(\tau(s)) x\left(t_{k}-h_{k}\right),
$$

where $\tilde{A}$ and $\tilde{A}_{d}$ are given in (6). Since those matrix functions are considered on $\tau(s) \in\left[0, T_{\max }\right]$, is continuous over this interval and are independent of the indice $k$, it is clear that there exist a constant parameter $\lambda^{*}$ such that $|x(s)| \leq \lambda^{*}\left|x_{t_{k}}\right|$. Since $\left|x_{t_{k}}\right|$ is converging to zero as $t_{k}$ tends to infinity, it is clear that $x(s)$ also converges to zero as $t_{k}$ tends to infinity.

A graphical illustration of Theorem 1 is shown in Figure 3 . The main idea remains in showing the equivalence between the conditions on the decreasing increment $\Delta V(k)=$ $V\left(x_{t_{k+1}}\right)-V\left(x_{t_{k}}\right)<0$ and the existence of a continuous functional $\mathcal{W}$ which coincides with the Lyapunov function $V$ at the sampling instants and which is strictly decreasing within all sampling intervals. The main contribution of Theorem 1 is that the introduction of the functional $\mathcal{V}$ allows the Lyapunov-Krasovskii functional $V$ to be locally increasing.

For the sake of simplicity, the notation $\tau, \boldsymbol{h}$ and $\boldsymbol{h}$ stand for the time-varying sampling delay $\tau(t)$, the time-varying transmission delay $h(t)$ and its time-varying derivative $\dot{h}(t)$.

\section{CASE of CONSTANT InPUT DELAY}

In this section, a study on the asymptotic stability of the solutions of sampled-data systems with constant input delay is provided. The objective is to design a functional which satisfies the conditions proposed in Theorem 1.

Theorem 2: For given delay $h>0$ and two positive scalar $T_{1}<T_{2}$, assume that there exist $Q>0, R_{1}>0$ and $R_{2}>$ $0 \in \mathbb{S}^{n}, P>0, U>0$ and $S_{1} \in \mathbb{S}^{2 n}$ and three matrices $S_{2}$ and $X \in \mathbb{R}^{2 n \times 2 n}, Y \in \mathbb{R}^{5 n \times 2 n}$ that satisfy for $i=1,2$

$$
\begin{gathered}
\Pi_{1}(h)+T_{i}\left(N_{2}^{T} X N_{2}+\Pi_{2}\right)<0, \\
{\left[\begin{array}{cc}
\Pi_{1}(h)-T_{i} N_{2}^{T} X N_{2} & T_{i} Y \\
T_{i} Y^{T} & -T_{i} U
\end{array}\right]<0,}
\end{gathered}
$$


where

$$
\begin{aligned}
& \Pi_{1}(h)=2 N_{1}^{T} P N_{0}-N_{12}^{T} S_{1} N_{12}-2 N_{2}^{T} S_{2} N_{12} \\
& \quad+M_{1}^{T} Q M_{1}-M_{2}^{T} Q M_{2}-M_{5}^{T} R_{1} M_{5} \\
& \quad+M_{0}^{T}\left(R_{1}+h R_{2}\right) M_{0}-M_{12}^{T} R_{2} / h M_{12}-2 Y N_{12} \\
& \Pi_{2}=N_{0}^{T}\left(U N_{0}+2 S_{1} N_{12}+2 S_{2}^{T} N_{2}\right),
\end{aligned}
$$

and

$$
\begin{aligned}
& M_{0}=\left[\begin{array}{lllll}
A & 0 & 0 & A_{d} & 0
\end{array}\right], M_{1}=\left[\begin{array}{lllll}
I & 0 & 0 & 0 & 0
\end{array}\right] \text {, } \\
& M_{2}=\left[\begin{array}{lllll}
0 & I & 0 & 0 & 0
\end{array}\right], M_{3}=\left[\begin{array}{lllll}
0 & 0 & I & 0 & 0
\end{array}\right] \text {, } \\
& M_{4}=\left[\begin{array}{lllll}
0 & 0 & 0 & I & 0
\end{array}\right], M_{5}=\left[\begin{array}{lllll}
0 & 0 & 0 & 0 & I
\end{array}\right] \\
& N_{0}=\left[M_{0}^{T} M_{5}^{T}\right]^{T}, N_{1}=\left[M_{1}^{T} M_{2}^{T}\right]^{T}, N_{2}=\left[M_{3}^{T} M_{4}^{T}\right]^{T} \text {, } \\
& M_{12}=M_{1}-M_{2}, N_{12}=N_{1}-N_{2}
\end{aligned}
$$

System (3) is thus asymptotically stable for any asynchronous sampling lying in $\left[T_{1}, T_{2}\right]$ and the delay $h$.

Proof: Consider the functional:

$$
\begin{aligned}
& V\left(t, x_{t}, \dot{x}_{t}\right)=y^{T}(t) P y(t)+\int_{t-h}^{t} x^{T}(s) Q x(s) d s \\
& +\int_{t-h}^{t} \dot{x}^{T}(s)\left(R_{1}+(h-t+s) R_{2}\right) \dot{x}(s) d s
\end{aligned}
$$

where $y(t)=\left[x^{T}(t) x^{T}(t-h)\right]^{T}$. Note that $V$ corresponds to a classical Lyapunov-Krasovskii functional type to cope with the stability of constant time-delay systems. The objective is here to ensure that the variation of the of $V$ between two successive sampling instant is negative. This means that $\Delta V=V\left(t_{k+1}, x_{t_{k+1}}, \dot{x}_{t_{k+1}}\right)-V\left(t_{k}, x_{t_{k}}, \dot{x}_{t_{k}}\right)$ is definite negative for all positive integer $k$. For any integer $k$ consider the additional functional

$$
\begin{aligned}
\mathcal{V}\left(t, \chi_{k}^{h}\right) & =\left(T_{k}-\boldsymbol{\tau}\right) \zeta_{0}^{T}(t)\left[S_{1} \zeta_{0}(t)+2 S_{2} y\left(t_{k}\right)\right] \\
& +\left(T_{k}-\boldsymbol{\tau}\right) \int_{t_{k}}^{t} \dot{y}^{T}(s) U \dot{y}(s) d s \\
& +\left(T_{k}-\boldsymbol{\tau}\right) \boldsymbol{\tau} y^{T}\left(t_{k}\right) X y\left(t_{k}\right)
\end{aligned}
$$

where $T_{k}$ lies in the interval $\left[T_{1}, T_{2}\right]$ and $\zeta_{0}(t)=y(t)-$ $y\left(t_{k}\right), \xi(s)=\left[y^{T}(s) y^{T}\left(t_{k}\right) \dot{x}^{T}(s-h)\right]^{T}$. The first step of the proof consists in ensuring condition (8). For $t=t_{k}$, $\zeta_{0}\left(t_{k}\right)=0$, the integral is zero and the last term is also zero since $\tau\left(t_{k}\right)=0$. When $t=t_{k+1}, T_{k}-\tau$ is equal to zero. Then (8) is satisfied. The functional $\mathcal{V}$ is thus continuous with respect to $t$ and equal to zero at all sampling instants and differentiable over $\left[t_{k} t_{k+1}[\right.$.

As suggested in the theorem, no additional constraint is introduced on $S_{1}, S_{2}, U$ and $X$ and $\mathcal{V}$ is not necessary positive definite within two sampling instants. This corresponds to the improvement with respect to the previous approaches exposed in [11], [15]. Note that the positivity of $U$ is not required but we will be introduced in the sequel.

The rest of the proof consists in ensuring $\dot{\mathcal{W}}<0$ over $\left[t_{k} t_{k+1}[\right.$. The computation of the derivative of $\dot{\mathcal{W}}$ leads to

$$
\begin{aligned}
\dot{\mathcal{W}}(t & \left., \chi_{k}^{h}\right)=2 y^{T}(t) P \dot{y}(t)+x^{T}(t) Q x(t) \\
& -x^{T}(t-h) Q x(t-h)+\dot{x}^{T}(t)\left(R_{1}+h R_{2}\right) \dot{x}(t) \\
& -\dot{x}^{T}(t-h) R_{1} \dot{x}(t-h)-\int_{t-h}^{t} \dot{x}^{T}(s) R_{2} \dot{x}(s) d s \\
& +\left(T_{k}-\tau\right) \dot{y}(t)^{T}\left[U \dot{y}(t)+2 S_{1} \zeta_{0}(t)+2 S_{2} y\left(t_{k}\right)\right] \\
& -\zeta_{0}^{T}(t)\left[S_{1} \zeta_{0}(t)+2 S_{2} y\left(t_{k}\right)\right] \\
& -\int_{t_{k}}^{t} \dot{y}^{T}(s) U \dot{y}(s) d s+\left(T_{k}-2 \tau\right) y^{T}\left(t_{k}\right) X y\left(t_{k}\right) .
\end{aligned}
$$

Applying Jensen inequality to the first integral, we have that

$$
\begin{aligned}
-\int_{t-h}^{t} & \dot{x}^{T}(s) R_{2} \dot{x}(s) d s \leq \\
& \quad(x(t)-x(t-h))^{T} R_{2} / h(x(t)-x(t-h))
\end{aligned}
$$

Consider a matrix $Y \in \mathbb{R}^{5 n \times 2 * n}$ and the following equality

$$
2 \xi^{T}(t) Y\left[y(t)-y\left(t_{k}\right)\right]=\int_{t_{k}}^{t}\left[2 \xi^{T}(t) Y \dot{y}(s)\right] d s .
$$

Since $U$ is assumed to be positive definite and thus non singular, a classical bounding ensures that for all $t \in\left[t_{k}, t_{k+1}[\right.$ and for all $s \in\left[t_{k}, t\right]$

$$
2 \xi^{T}(t) Y \dot{y}(s) \leq \xi^{T}(t) Y U^{-1} Y^{T} \xi(t)+\dot{y}^{T}(s) U \dot{y}(s) .
$$

Integrating the previous inequality over $\left[t_{k}, t\right]$, the following inequality is obtained

$$
\begin{aligned}
-\int_{t_{k}}^{t} \dot{y}^{T}(s) U \dot{y}(s) d s \leq & -2 \xi^{T}(t) Y\left(y(t)-y\left(t_{k}\right)\right) \\
& +\boldsymbol{\tau} \xi^{T}(t) Y U^{-1} Y^{T} \xi(t) .
\end{aligned}
$$

Noting that

$$
\begin{aligned}
& \dot{x}(t)=A x(t)+A_{d} x\left(t_{k}\right)=M_{0} \xi(t), \\
& x(t)=M_{1} \xi(t), \quad x(t-h)=M_{2} \xi(t), \\
& x(t)-x(t-h)=M_{12} \xi(t), \quad y(t)=N_{1} \xi(t), \\
& y\left(t_{k}\right)=N_{2} \xi(t), \quad \zeta_{0}(t)=y(t)-y\left(t_{k}\right)=N_{12} \xi(t) \\
& \dot{y}(t)=\left[\left(A x(t)+A_{d} x\left(t_{k}\right)\right)^{T} \quad \dot{x}^{T}(t-h)\right]^{T}=N_{0} \xi(t)
\end{aligned}
$$

and substituting (16) to (14), the following inequality is obtained for all $t \in\left[t_{k}, t_{k+1}[\right.$

$$
\begin{aligned}
\dot{\mathcal{W}}\left(t, \chi_{k}^{h}\right) & \leq \xi^{T}(t)\left[\Pi_{1}(h)+\left(T_{k}-\tau\right) \Pi_{2}\right. \\
& \left.+\left(T_{k}-2 \tau\right) N_{2}^{T} X N_{2}+\tau N U^{-1} N^{T}\right] \xi(t) .
\end{aligned}
$$

Based on a convexity argument on $\tau$, the right hand-side term is negative definite if and only if

$$
\Pi_{1}(h)+T_{k}\left(\Pi_{2}+N_{2}^{T} X N_{2}\right)<0,
$$

and

$$
\Pi_{1}(h)+T_{k}\left(Y U^{-1} Y^{T}-N_{2}^{T} X N_{2}\right)<0 .
$$

Using the same convexity argument on $T_{k} \in\left[T_{1}, T_{2}\right]$, the LMI's (10) and (11) are retrieved. By virtue of Theorem 1, the asymptotically stability of system (3) is guaranteed.

Note that the conditions from Theorem 2 include the robust stability properties with respect to the input delay $h$. This means that (10) and (11) require the system to be stable at least for the transmission delay $h$ and $T_{1}=T_{2}=0$.

\section{CASE OF TIME-VARYING INPUT DELAY}

Consider that input delay $h$ is now time-varying and satisfies (2). In the sequel the notations $h$ and $\dot{h}$ stands for the time-varying delay $h(t)$ and its time-varying derivative $\dot{h}(t)$. Since the transmission is time-varying, the samplings at the actuator and at the sensor are not the same. Then it the sequel, the stability criteria focuses on the sampling at the actuator denote by $\bar{T}_{k} \in\left[\bar{T}_{1}, \bar{T}_{2}\right]$. The following theorem is proposed:

Theorem 3: Consider a time-varying delay $h$ which satisfies (2) and two positive scalar $0 \leq \bar{T}_{1}<\bar{T}_{2}$, assume that there exist $Q>0, R_{1}>0$ and $R_{2}>0 \in \mathbb{S}^{n}, P>0, U>0$ 
and $S_{1} \in \mathbb{S}^{2 n}$ and three matrices $S_{2}$ and $X \in \mathbb{R}^{2 n \times 2 n}, Y_{1}$ and $Y_{2} \in \mathbb{R}^{5 n \times 2 n}$ that satisfy for $i=1,2$ and $j=1,2$

$$
\begin{gathered}
{\left[\begin{array}{cc}
\Pi_{3}\left(h_{j}\right)+\bar{T}_{i}\left(N_{2}^{T} X N_{2}+\Pi_{2}\right) & \left(1-\epsilon_{2}\right) h_{j} Y_{2} \\
\left(1-\epsilon_{2}\right) h_{j} Y_{2}^{T} & -\left(1-\epsilon_{2}\right) h_{j} R_{2}
\end{array}\right]<0,} \\
{\left[\begin{array}{ccc}
\Pi_{3}\left(h_{i}\right)-\bar{T}_{i} N_{2}^{T} X N_{2} & \bar{T}_{i} Y_{1} & \left(1-\epsilon_{2}\right) h_{j} Y_{2} \\
\bar{T}_{i} Y_{1}^{T} & -\bar{T}_{i} U & 0 \\
\left(1-\epsilon_{2}\right) h_{j} Y_{2}^{T} & 0 & -\left(1-\epsilon_{2}\right) h_{j} R_{2}
\end{array}\right]<0,}
\end{gathered}
$$

where $\Pi_{2}$ and the matrices $N_{i}$ and $M_{i}$ are given in Theorem 2 and

$$
\begin{aligned}
\Pi_{3}\left(h_{j}\right) & =2 N_{1}^{T} P N_{0}-N_{12}^{T} S_{1} N_{12}-2 N_{2}^{T} S_{2} N_{12} \\
& +M_{1}^{T} Q M_{1}-\left(1-\epsilon_{2}\right) M_{2}^{T} Q M_{2} \\
& +M_{0}^{T}\left(R_{1}+h_{j} R_{2}\right) M_{0}-1 /\left(1-\epsilon_{1}\right) M_{5}^{T} R_{1} M_{5} \\
& -2 Y_{1} N_{12}-2\left(1-\epsilon_{2}\right) Y_{2} M_{12},
\end{aligned}
$$

System (3) is thus asymptotically stable for the sampling period $T$ and the time-varying input delay $h$.

Proof: Consider the same functional as defined in Theorem 2 but with a time-varying delay $h(t)$ :

$$
\begin{aligned}
V\left(t, x_{t}, \dot{x}_{t}\right)=z^{T}(t) P z(t)+\int_{t-\boldsymbol{h}}^{t} x^{T}(s) Q x(s) d s \\
+\int_{t-\boldsymbol{h}}^{t} \dot{x}^{T}(s)\left(R_{1}+(\boldsymbol{h}-t+s) R_{2}\right) \dot{x}(s) d s
\end{aligned}
$$

where $z=\left[x^{T}(t) x^{T}(t-h)\right]^{T}$ and the additional functional $\mathcal{V}$

$$
\begin{aligned}
\mathcal{V}\left(t, \chi_{k}^{h_{2}}\right) & =\left(\bar{T}_{k}-\boldsymbol{\tau}\right) \zeta_{1}^{T}(t)\left[S_{1} \zeta_{1}(t)+2 S_{2} z\left(t_{k}\right)\right] \\
& +\left(\bar{T}_{k}-\boldsymbol{\tau}\right) \int_{t_{k}}^{t} \dot{z}^{T}(s) U \dot{z}(s) d s \\
& +\left(\bar{T}_{k}-\boldsymbol{\tau}\right) \boldsymbol{\tau} z^{T}\left(t_{k}\right) X z\left(t_{k}\right)
\end{aligned}
$$

where $\zeta_{1}(t)=z(t)-z\left(t_{k}\right), \phi(s)=\left[z^{T}(s) z^{T}\left(t_{k}\right)(1-\right.$ $\left.\dot{\boldsymbol{h}}) \dot{x}^{T}(t-\boldsymbol{h})\right]^{T}$. Following the proof of Theorem 2, we prove that the functional $\mathcal{V}$ satisfies (8), is continuous with respect to the time variable and $\mathcal{W}$ and $V$ coincide at all sampling instants. The computation of the derivative of $\dot{\mathcal{W}}$ leads to

$$
\begin{aligned}
& \dot{\mathcal{W}}\left(t, \chi_{k}^{h_{2}}\right)=2 z^{T}(t) P \dot{z}(t)+x^{T}(t) Q x(t)+\dot{x}^{T}(t) R_{1} \dot{x}(t) \\
& \quad+\boldsymbol{h} \dot{x}^{T}(t) R_{2} \dot{x}(t)-(1-\dot{\boldsymbol{h}}) x^{T}(t-\boldsymbol{h}) Q x(t-\boldsymbol{h}) \\
& \quad-(1-\dot{\boldsymbol{h}})\left[\dot{x}^{T}(t-\boldsymbol{h}) R_{1} \dot{x}(t-\boldsymbol{h})+\int_{t-\boldsymbol{h}}^{t} \dot{x}^{T}(s) R_{2} \dot{x}(s) d s\right] \\
& \quad+\left(\bar{T}_{k}-\boldsymbol{\tau}\right) \dot{z}(t)^{T}\left[U \dot{z}(t)+2 S_{1} \zeta_{1}(t)+2 S_{2} z\left(t_{k}\right)\right] \\
& \quad-\zeta_{1}^{T}(t)\left[S_{1} \zeta_{1}(t)+2 S_{2} z\left(t_{k}\right)\right]-\int_{t_{k}}^{t} \dot{z}^{T}(s) U \dot{z}(s) d s \\
& \quad+\left(\bar{T}_{k}-2 \boldsymbol{\tau}\right) z^{T}\left(t_{k}\right) X z\left(t_{k}\right) .
\end{aligned}
$$

From the definition of the delay $\boldsymbol{h}$ given in (2), and knowing that the matrices $Q, R_{1}$ and $R_{2}$ are positive definite, we have $-(1-\dot{\boldsymbol{h}}) \leq-\left(1-\epsilon_{2}\right)$ and

$$
\begin{aligned}
-(1-\dot{\boldsymbol{h}}) & \dot{x}^{T}(t-\boldsymbol{h}) R_{1} \dot{x}(t-\boldsymbol{h}) \\
& \leq-1 /(1-\dot{\boldsymbol{h}}) \phi^{T}(t) M_{5}^{T} R_{1} M_{5} \phi(t) \\
& \leq-1 /\left(1-\epsilon_{1}\right) \phi^{T}(t) M_{5}^{T} R_{1} M_{5} \phi(t)
\end{aligned}
$$

For any matrix $Y_{2} \in \mathbb{R}^{5 n \times 2 n}$, the first integral of (22) is bounded as follows

$$
\begin{aligned}
-\int_{t-\boldsymbol{h}}^{t} \dot{x}^{T}(s) R_{2} \dot{x}(s) d s & \leq-2 Y_{2}(x(t)-x(t-\boldsymbol{h})) \\
& +\boldsymbol{h} \phi^{T}(t) Y_{2} R_{2}^{-1} Y_{2}^{T} \phi(t),
\end{aligned}
$$

and for any matrix $Y_{1} \in \mathbb{R}^{5 n \times 2 n}$ and for all $t \in\left[t_{k}, t_{k+1}[\right.$, the following inequality is obtained

$$
\begin{aligned}
-\int_{t_{k}}^{t} \dot{z}^{T}(s) U \dot{z}(s) d s \leq & -2 \xi^{T}(t) Y_{1}\left(z(t)-z\left(t_{k}\right)\right) \\
& +\boldsymbol{\tau} \phi^{T}(t) Y_{1} U^{-1} Y_{1}^{T} \phi(t),
\end{aligned}
$$

Using the definition of the matrices $M_{i}$ 's and $N_{i}$ 's and using the previous inequality into (22), the following inequality is obtained for all $t \in\left[t_{k}, t_{k+1}[\right.$

$$
\begin{aligned}
\dot{\mathcal{W}}\left(t, \chi_{k}^{h_{2}}\right) \leq & \phi^{T}(t)\left[\Pi_{1}(\boldsymbol{h})+\left(\bar{T}_{k}-\boldsymbol{\tau}\right) \Pi_{2}\right. \\
& +\left(\bar{T}_{k}-2 \boldsymbol{\tau}\right) N_{2}^{T} X N_{2}+\boldsymbol{\tau} Y_{1} U^{-1} Y_{1}^{T} \\
& \left.+\boldsymbol{h} Y_{2} R_{2}^{-1} Y_{2}^{T}\right] \phi(t) .
\end{aligned}
$$

Applying a convexity argument on $\tau$, the right hand-side term is negative definite if and only if

$$
\begin{gathered}
\Pi_{1}(\boldsymbol{h})+\bar{T}_{k}\left(\Pi_{2}+N_{2}^{T} X N_{2}\right)+\boldsymbol{h} Y_{2} R_{2}^{-1} Y_{2}^{T}<0, \\
\Pi_{1}(\boldsymbol{h})+\bar{T}_{k}\left(N U^{-1} N^{T}-N_{2}^{T} X N_{2}\right)+\boldsymbol{h} Y_{2} R_{2}^{-1} Y_{2}^{T}<0 .
\end{gathered}
$$

Then Noting that the previous conditions depend linearly on $h$ and on $\bar{T}_{k}$, we apply once more a convexity argument on this two variables. The Schur complement allows obtaining conditions (18) and (19). By virtue of Theorem 1, the asymptotically stability of system (3) is guaranteed.

If the minimal bound of the transmission delay $h_{1}$ is zero and $T_{1} \leq h_{2}$, the lower bound of the sampling period at the actuator is $\bar{T}_{1}=0$ from equation (5). Then the previous theorem can be simplified into the following corollary.

Corollary 1: If $h_{1}=0$, the conditions (18) and (19) become

$$
\Pi_{1}(0)<0, \quad \Pi_{1}(0)+\bar{T}_{2}\left(N_{2}^{T} X N_{2}+\Pi_{2}\right)<0,
$$

$$
\left[\begin{array}{cc}
\Pi_{1}\left(h_{2}\right) & \left(1-\epsilon_{2}\right) h_{2} Y_{2} \\
\left(1-\epsilon_{2}\right) h_{2} Y_{2}^{T} & -\left(1-\epsilon_{2}\right) h_{2} R_{2}
\end{array}\right]<0
$$

$\left[\begin{array}{ccc}\Pi_{1}\left(h_{2}\right)-\bar{T}_{2} N_{2}^{T} X N_{2} & \bar{T}_{2} Y_{1} & \left(1-\epsilon_{2}\right) h_{2} Y_{2} \\ \bar{T}_{2} Y_{1}^{T} & -\bar{T}_{2} U & 0 \\ \left(1-\epsilon_{2}\right) h_{2} Y_{2}^{T} & 0 & -\left(1-\epsilon_{2}\right) h_{2} R_{2}\end{array}\right]<0$

Remark 1: It is clear that discrete-time approaches proposed for instance in [5] or in [10] leads to less conservative stability conditions. However it is possible to extend those stability criteria to the case of polytopic uncertainties. Since all the stability conditions provided in this article are linear with respect to the system matrices $A$ and $A_{d}$, it is possible to extend Theorems 2 and 3 to cop with parameter uncertainties. This makes the proposed method still relevant with respect to the discrete-time approach.

\section{EXAMPLES}

- Example 1 [3], [14]

$$
\dot{x}(t)=\left[\begin{array}{cc}
0 & 1 \\
0 & -0.1
\end{array}\right] x(t)+\left[\begin{array}{cc}
0 & 0 \\
-0.375 & -1.15
\end{array}\right] x\left(s_{k}\right) .
$$

- Example 2 [2]

$$
\dot{x}(t)=\left[\begin{array}{cc}
-2 & 0 \\
0 & -0.9
\end{array}\right] x(t)+\left[\begin{array}{cc}
-1 & 0 \\
-1 & -1
\end{array}\right] x\left(s_{k}\right)
$$

The maximal allowable sampling period are summarized in Table I for time-varying sampling and constant delay and in Table II for time-varying delay. 


\begin{tabular}{|c|c|c|c|c|c|c|c|}
\hline Ex.1 $h_{2}$ & $10^{-3}$ & 0.2 & 0.4 & 0.6 & 0.8 & 1 & 1.075 \\
\hline$[15]$ & 1.111 & 0.714 & 0.469 & 0.269 & 0.069 & - & - \\
{$[12]$} & 1.043 & 0.846 & 0.650 & 0.456 & 0.262 & 0.071 & - \\
{$[11]$} & 1.638 & 1.063 & 0.786 & 0.541 & 0.301 & 0.054 & - \\
\hline Th. 2 & 1.717 & 1.435 & 1.149 & 0.858 & 0.540 & 0.167 & $10^{-3}$ \\
\hline \hline Ex.2 $h_{2}$ & $10^{-3}$ & 0.5 & 1 & 2 & 3 & 4 & 4.472 \\
\hline$[15]$ & 1.278 & 0.878 & - & - & - & - & - \\
{$[12]$} & 1.867 & 1.380 & 1.042 & 0.608 & 0.314 & 0.090 & $10^{-3}$ \\
{$[11]$} & 1.970 & 1.368 & 0.868 & 0.212 & 0.038 & - & - \\
\hline \hline Th. 2 & 2.624 & 2.126 & 1.929 & 1.530 & 1.118 & 0.605 & $10^{-3}$ \\
\hline
\end{tabular}

TABLE I

MAXIMAL SAMPLING PERIOD AT THE ACTUATOR $T$ FOR SEVERAL CONSTANT DELAY USING THEOREM 2 FOR EXAMPLES 1 AND 2.

\begin{tabular}{|c|c|c|c|c|c|c|c|}
\hline Ex.1 $h_{2}$ & $10^{-3}$ & 0.2 & 0.4 & 0.6 & 0.8 & 1 & 1.075 \\
\hline$[15]$ & 1.111 & 0.804 & 0.544 & 0.296 & 0.070 & - & - \\
{$[12]$} & 1.042 & 0.843 & 0.643 & 0.443 & 0.243 & 0.043 & - \\
{$[11]$} & 1.638 & 1.063 & 0.786 & 0.541 & 0.301 & 0.054 & - \\
\hline Th. $3^{1}$ & 1.670 & 1.209 & 0.994 & 0.760 & 0.479 & 0.095 & - \\
Th. $3^{2}$ & 1.658 & 1.084 & 0.718 & 0.203 & - & - & - \\
\hline \hline Ex.2 $h_{2}$ & $10^{-3}$ & 0.5 & 1 & 2 & 3 & 4 & 4.472 \\
\hline$[15]$ & 1.278 & 0.575 & - & - & - & - & - \\
{$[12]$} & 1.867 & 1.368 & 0.868 & - & - & - & - \\
{$[11]$} & 1.970 & 1.368 & 0.868 & 0.443 & 0.212 & 0.038 & - \\
\hline \hline Th. $3^{1}$ & 2.407 & 1.770 & 1.488 & 1.010 & 0.179 & - & - \\
Th. $3^{2}$ & 2.387 & 1.590 & 1.228 & - & - & - & - \\
\hline
\end{tabular}

TABLE II

MAXIMAL SAMPLING PERIOD AT THE ACTUATOR $\bar{T}$ FOR SEVERAL TIME-VARYING TRANSMISSION DELAYS USING THEOREM3 FOR EXAMPLES 1 AND 2.

Consider the case of constant transmission delays indicated obtained by solving Theorem 2 using $\bar{T}_{1}=T_{1}=0$. It can be seen in Table I that it delivers less conservative results than the existing ones based on a continuous time-approach.

Concerning the time-varying transmission delay case, we consider $h_{1}=10^{-4}, \bar{T}_{1}=0, \epsilon_{1}=-0.2$ and the two case $\epsilon_{2}=0.5$ and 0.8 , respectively denoted by the superscripts ${ }^{1}$ and ${ }^{2}$ in Table II. They show the influence of the delay variation $\epsilon_{2}$. The influence of $\epsilon_{1}$ is not presented here because it is not as relevant as the one of $\epsilon_{2}$. First, we can see that, for small value of $\epsilon_{2}$, here $\epsilon_{2}=0.5$, the result are less conservative than existing once. However for larger values of $\epsilon_{2}$, i.e. $\epsilon_{2}=0.8$, the maximum allowable sampling period obtained by solving the Theorem 3 still delivers less conservative results than the existing ones for small values of $h_{2}$. However Theorem 3 becomes more conservative when $h_{2}$ is larger. Note that in [9], stability conditions based on Theorem 1 have been improved to avoid the dependence on the derivative of the transmission delays, i.e. on the $\epsilon_{i}$ 's.

\section{CONCLUSION}

An novel analysis of NCS under asynchronous sampling and input delay is provided in this article. This approach is based on the discrete-time Lyapunov Theorem applied to the continuous-time model of the NCS. Tractable conditions are derived to ensure asymptotic stability. The examples show the efficiency of the method and the reduction of the conservatism compared to other results from the literature.

\section{REFERENCES}

[1] T. Chen and B.A. Francis. Optimal sampled-data control systems. Springer-Verlag, Berlin, Germany, 1995.

[2] E. Fridman. A refined input delay approach to sampled-data control. Automatica, 46(2):421-427, 2010.

[3] E. Fridman, A. Seuret, and J.-P. Richard. Robust sampled-data stabilization of linear systems: An input delay approach. Automatica, 40(8):1141-1446, 2004.

[4] E. Fridman and U. Shaked. Stability and guaranteed cost control of uncertain discrete delay systems. International Journal of Control, 45(12):2406-2411, 2000

[5] H. Fujioka. Stability analysis of systems with aperiodic sample-andhold devices. Automatica, 45(3):771-775, 2009.

[6] K. Gu, V.-L. Kharitonov, and J. Chen. Stability of time-delay systems. Birkhauser, 2003.

[7] J.P. Hespanha, P. Naghshtabrizi, and Y. Xu. A survey of recent results in networked control systems. Proceedings of the IEEE, 95(1):138$162,2007$.

[8] L. Hetel, J. Daafouz, and C. Iung. Stabilization of arbitrary switched linear systems with unknown time-varying delays. Automatic Control, IEEE Transactions on, 51(10):1668-1674, Oct. 2006.

[9] W. Jiang and A. Seuret. Improved stability analysis of networked control systems under asynchronous sampling and input delay. In $2^{\text {nd }}$ IFAC Workshop on Distributed Control and Estimation of Networked control systems, September 13-14 2010.

[10] X.-G. li, A. Çela, S.-I. Niculescu, and A. Reama. Some remark on the stability of networked control systems with periodic scheduling. In Proceedings of the European Control Conference, Budapest, Hongary, 2009.

[11] K. Liu and E. Fridman. Stability analysis of networked control systems: a discontiuous Lyapunov functional approach. In Joint $48^{\text {th }}$ IEEE Conference on Decision and Control and $28^{\text {th }}$ Chinese Control Conference, Shanghai, China, 2009.

[12] P. Millán, L. Orihuela, C. Vivas, and F.R. Rubio. Improved delaydependent stability criterion for uncertain networked control systems with induced time-varying delays. In $1^{\text {rst }}$ IFAC Workshop on Distributed estimation and Control of Networked control Systems, Necsys'09, Venise, Italy, 2009.

[13] L. Mirkin. Some remarks on the use of time-varying delay to model sample-and-hold circuits. IEEE Trans. on Automatic Control, 52(6):1009-1112, 2007.

[14] P. Naghshtabrizi, J.P. Hespanha, and A.R. Teel. Exponential stability of impulsive systems with application to uncertain sampled-data systems. Systems and Control Letters, 57(5):378-385, 2008.

[15] P. Naghshtabrizi, J.P. Hespanha, and A.R. Teel. Stability of delay impulsive systems with application to networked control systems. Trans. of the Inst. of Measurement and Control, Special Issue on Hybrid and Switched Systems, 2009.

[16] Y. Oishi and H. Fujioka. Stability and stabilization of aperiodic sampled-data control systems using robust linear matrix inequalities. Automatica, 46(8):1327-1333, 2010.

[17] M.M. Peet, A. Papachristodoulou, and S. Lall. Positive forms and stability of linear time-delay systems. SIAM Journal on Control and Optimization, 47(6):3227-3258, 2009.

[18] J.-P. Richard. Time delay systems: an overview of some recent advances and open problems. Automatica, 39:1667-1694, 2003.

[19] A. Seuret. Stability analysis for sampled-data systems with a timevarying period. In $48^{\text {th }}$ IEEE Conference on Decision and Control, December 2009.

[20] A. Seuret. A novel stability analysis of sampled-data systems with applications to multi-rate sampling and packet loss. submitted to Automatica, 2010.

[21] D. Yue, Q.-L. Han, and J. Lam. Stability and stabilization of nonuniform sampling systems. Automatica, 44(12):3222-3226, 2008.

[22] S. Zampieri. A survey of recent results in Networked Control Systems. In Proc. $17^{\text {th }}$ IFAC World Congress, Seoul, Korea, July 2008.

[23] W. Zhang and M.S. Branicky. Stability of networked control systems with time-varying transmission period. In Allerton Conf. Communication, Control, and Computing, October 2001.

[24] W. Zhang, M.S. Branicky, and S.M. Phillips. Stability of networked control systems. IEEE Control Systems Magazine, (21), 2001. 